\title{
Exciton Drift in Semiconductors under Uniform Strain Gradients: Application to Bent $\mathrm{ZnO}$ Microwires
}

\author{
Xuewen Fu, ${ }^{\dagger}$ Gwenole Jacopin, ${ }^{\ddagger}$ Mehran Shahmohammadi, ${ }^{\ddagger}$ Ren Liu, ${ }^{\dagger}$ Malik Benameur, ${ }^{\S}$ \\ Jean-Daniel Ganière, ${ }^{\ddagger}$ Ji Feng, ${ }^{\perp}$ Wanlin Guo," Zhi-Min Liao, ${ }^{\dagger}$ Benoit Deveaud, ${ }^{\neq, *}$ and Dapeng $\mathrm{Yu}^{\dagger, *}$ \\ ${ }^{\dagger}$ State Key Laboratory for Mesoscopic Physics, and Electron Microscopy Laboratory, Department of Physics, Peking University, 209 Chengfu Road, Beijing 100871, \\ China, ${ }^{\ddagger}$ Laboratoire d'Optoélectronique Quantique, École Polytechnique Fédérale de Lausanne (EPFL), Station 3, CH-1015 Lausanne, Switzerland, ${ }^{\S}$ Attolight AG, \\ EPFL Innovation Square, PSE D, 1015 Lausanne, Switzerland, ${ }^{\perp}$ International Center for Quantum Materials, School of Physics, Peking University, Beijing 100871, \\ China, and "State Key Laboratory of Mechanics and Control of Mechanical Structures and Institute of Nano Science, Nanjing University of Aeronautics and \\ Astronautics, 29 Yudao Street, Nanjing 210016, China
}

\begin{abstract}
Optimizing the electronic structures and carrier dynamics in semiconductors at atomic scale is an essential issue for innovative device applications. Besides the traditional chemical doping and the use of homo/heterostructures, elastic strain has been proposed as a promising possibility. Here, we report on the direct observation of the dynamics of exciton transport in a $\mathrm{ZnO}$ microwire under pure elastic bending deformation, by using cathodoluminescence with high temporal, spatial, and energy resolutions. We demonstrate that excitons can be effectively drifted by the strain gradient in inhomogeneous strain fields. Our observations are well reproduced by a drift-diffusion model taking into account the strain gradient and allow us to deduce an exciton mobility of $1400 \pm 100 \mathrm{~cm}^{2} /(\mathrm{eV} \mathrm{s})$ in the $\mathrm{Zn} 0$ wire. These results propose a way to tune the exciton dynamics in semiconductors and imply the possible role of strain gradient in optoelectronic and sensing nano/microdevices.
\end{abstract}

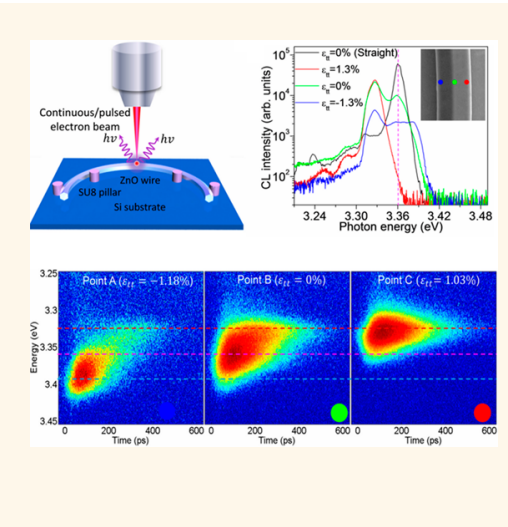

KEYWORDS: ZnO microwire $\cdot$ time-resolved cathodoluminescence $\cdot$ pure bending strain $\cdot$ exciton dynamics $\cdot$ strain gradient

\begin{abstract}
$t$ is essential for new concept devices, in particular for very small-scale devices, to be able to optimize the electronic structure and carrier dynamics of semiconductors at the atomic scale. Following a classical paradigm, chemical doping allows constructing specially designed electronic band structures, such as quantum wells and homo/heterojunctions. It is widely used in innumerable applications such as optical detection, ${ }^{1,2}$ photovoltaics, ${ }^{3,4}$ semiconductor lasers, ${ }^{5}$ and light-emitting diodes. ${ }^{6}$ Elastic strain, with the exceptional ability to tune the lattice parameter continuously and reversibly, has also been proposed as a possible knob, allowing to modulate the electronic structure and carrier dynamics in semiconductors. ${ }^{7-9}$ Notable examples are the strained silicon technology for enhancing carrier mobility in advanced transistors $^{10}$ and piezoelectricity in polar semiconductors. ${ }^{11,12}$ The field is bound to become even more prominent through the recent development of semiconducting
\end{abstract}

micro/nanostructures, which possess, thanks to their high surface to volume ratio, much higher mechanical toughness and strength compared to their bulk counterparts. ${ }^{13,14}$ Because the elastic strain effects are greatly magnified at the micro/nanoscale, it is possible to significantly tune their properties by elastic deformation, staying away from inelastic relaxation via plasticity or rupture. ${ }^{7}$

Recently, major progress has been achieved on elastic strain engineered semiconductor micro/nanostructures. For instance, remarkable red energy shifts of the near-band-edge (NBE) luminescence have been observed in uniaxially strained $\mathrm{GaAs}^{15}$ and $\mathrm{ZnO}^{16}$ nanowires, as well as in bent $\mathrm{ZnO}^{17-21}$ and $\mathrm{CdS}^{22,23} \mathrm{micro} /$ nanowires through photoluminescence (PL) or cathodoluminescence (CL). Particularly, due to the coupling of piezoelectric and semiconducting properties, bending deformation in $\mathrm{ZnO}$ micro/nanowires has given rise to newfangled nanogenerators ${ }^{13}$ and piezo-phototronic devices. ${ }^{24}$ In all these

\section{* Address correspondence to benoit.deveaud-pledran@epfl.ch; yudp@pku.edu.cn.}

Received for review December 4, 2013 and accepted March 21, 2014.

Published online

10.1021/nn4062353

C XXXX American Chemical Society 
strained micro/nanostructure designs, the strain gradient is exceptionally large and plays an important role.

Even if the use of deformation potentials to control the carrier mobility has been proposed for decades, $^{25-27}$ the influence of an elastic inhomogeneous strain field on the carrier dynamics in semiconductors is however still elusive. Indeed, due to the difficulty in precisely creating and controlling an elastic inhomogeneous strain field in micro/nanostructures and the lack of an experimental characterization technique with enough spatial and temporal resolutions, no direct experimental observation of the dynamics of excitons under a well-defined strain gradient has yet been reported. Here, we investigated exciton dynamics in a purely bent $\mathrm{ZnO}$ microwire (MW) with a uniform strain gradient by both continuous-wave $\mathrm{CL}$ (CW-CL) and picosecond time-resolved CL (pTRCL) techniques (with spatial resolution limited by the size of the generation volume of around $200 \mathrm{~nm}$ and temporal resolution of a few picoseconds). The local optical properties of the wire were examined at both low $(8 \mathrm{~K})$ and room temperatures.

We directly observe that the donor-bound excitons can be effectively drifted by the uniform elastic strain gradient in a purely bent $\mathrm{ZnO} \mathrm{MW}$ of high quality. The excitons move toward the outer tensile side of the bent section, because of the continuous variation of the band gap (thus of the exciton energy) induced by the bending deformation. A drift-diffusion model allows to describe accurately the exciton dynamics in the inhomogeneous strain field and reproduces well the experimental results. The model allows us to deduce a high mobility of $1400 \pm 100 \mathrm{~cm}^{2} \mathrm{eV}^{-1} \mathrm{~s}^{-1}$ for the donor-bound excitons in the $\mathrm{ZnO} \mathrm{MWs}$.

\section{RESULTS AND DISCUSSION}

We use hexagonal $\mathrm{ZnO} \mathrm{MWs}$ oriented along the [0001] direction, with a diameter ranging between 1 and $3 \mu \mathrm{m}$, grown by chemical vapor deposition (CVD) (see Materials and Methods section). To produce, in a simple way, a precisely adjustable inhomogeneous deformation, we utilize a four-point-bending (4PB) configuration, ${ }^{28}$ as schematically shown in Figure $1 \mathrm{a}$. In this 4PB configuration, the wire is separated into five different segments subjected to different strains. The middle segment, which we are studying here, shows a well-defined pure bending state with uniform strain gradient. The pure bending deformation is controlled precisely by carefully designing the configuration of the constraining pillars according to ASTM E855-08 specification. ${ }^{29}$ The local strain $\varepsilon_{\mathrm{tt}}$ along the [0001] axis changes linearly from compression (inside the MW) to tension (outside the MW), passing through a strainneutral plane (i.e., the geometric neutral plane). The strain varies as $\varepsilon_{\mathrm{tt}}=r / \rho(|r \leq d / 2|)$, which leads to a constant strain gradient $\left(\partial \varepsilon_{\mathrm{tt}} / \partial r=1 / \rho\right)$ along the wire cross-section, $\rho$ being the radius of curvature of the strain-neutral plane, $r$ the distance relative to the strainneutral plane, and $d$ the diameter of the wire (see Figure 1b). Figure 1a schematically depicts both the CW-CL and PTRCL measurements in the pure bending region of such a $4 \mathrm{~PB} \mathrm{ZnO} M W$. Figure $1 \mathrm{c}$ shows a typical SEM image of a 4PB ZnO MW. The accurate circular shape of the MW (dotted line in Figure 1c) confirms the pure bending deformation. The maximum values of the tensile and compressive strains at the outer and inner edges are estimated to be $\varepsilon_{\mathrm{tt}}^{\max }= \pm 1.40 \%$. Importantly enough, the $\mathrm{ZnO} \mathrm{MW}$ is elastically deformed, as it returns fully to the initial straight geometry once the constraints are released.

We first performed $\mathrm{CW}-\mathrm{CL}$ line scanning across this $4 \mathrm{~PB} \mathrm{ZnO} \mathrm{MW}$ at $8 \mathrm{~K}$ (see Materials and Methods section). The line scanning $\mathrm{CW}-\mathrm{CL}$ spectra collected at both the straight and pure bending sections (indicated by the red ellipses " $\mathrm{I}$ " and "II" in Figure 1C) are presented in Figure $1 d$ and $f$, respectively. The $C L$ spectra are very sharp, evidencing the quality of the wire. More precisely, the $\mathrm{CL}$ spectrum of the straight segment shows a dominant transition from neutral donor-bound exciton (3.360 eV, $\mathrm{D}^{0} \mathrm{X}_{\mathrm{A}}$ ), with additionally the two-electron satellite (3.324 eV, TES) and the LO phonon replica of $D^{0} X_{A}(3.292 \mathrm{eV}) .^{30,31}$ There is no change when moving the spot across the MW (see Figure 1d). In the pure bending segment, on the contrary, the luminescence spectrum depends on the excitation position (see Figure $1 \mathrm{f}$ ). It is dominated by a red-shifted peak (3.322 eV) compared to the $D^{0} X_{A}$ line in the unstrained wire. Two other weak but welldefined peaks emerge at higher energy when the excitation spot is moved toward the compressive side. The highest energy peak continuously blue shifts, while the energy of the middle one remains almost unchanged. The corresponding CW-CL spectra recorded at three locations (outer side, middle, and inner side) across the bent section and one in the unstrained segment are displayed in Figure 1e.

The spectra obtained under CW-CL mode at room temperature, on the same 4PB specimen, show some significant differences. At room temperature, the results obtained (Supplementary Figure S2) compare well with the previous studies, ${ }^{18-20}$ i.e., a linear blueshift in the compressive side and red-shift in the tensile side of bent $\mathrm{ZnO}$ micro/nanowires. From the curvature of the wire estimated from the SEM image, we can deduce a deformation potential $\left(\partial E_{\mathrm{ex}} / \partial \varepsilon_{\mathrm{tt}}\right)$ of around $-27.3 \mathrm{meV} / \%$ at room temperature (Supplementary Figure S2d). This value is larger than Dietrich's experimental result (about $-20.4 \mathrm{meV} / \%)^{18}$ on bent $\mathrm{ZnO}$ MWs and close to Rowe's experimental result (about $-29.5 \mathrm{meV} / \%)^{32}$ on homogeneously stressed $\mathrm{ZnO}$. The difference, between $8 \mathrm{~K}$ and room temperature, of the NBE luminescence features within the pure bending section is probably due to the coupling between strained lattice and exciton dynamics. 

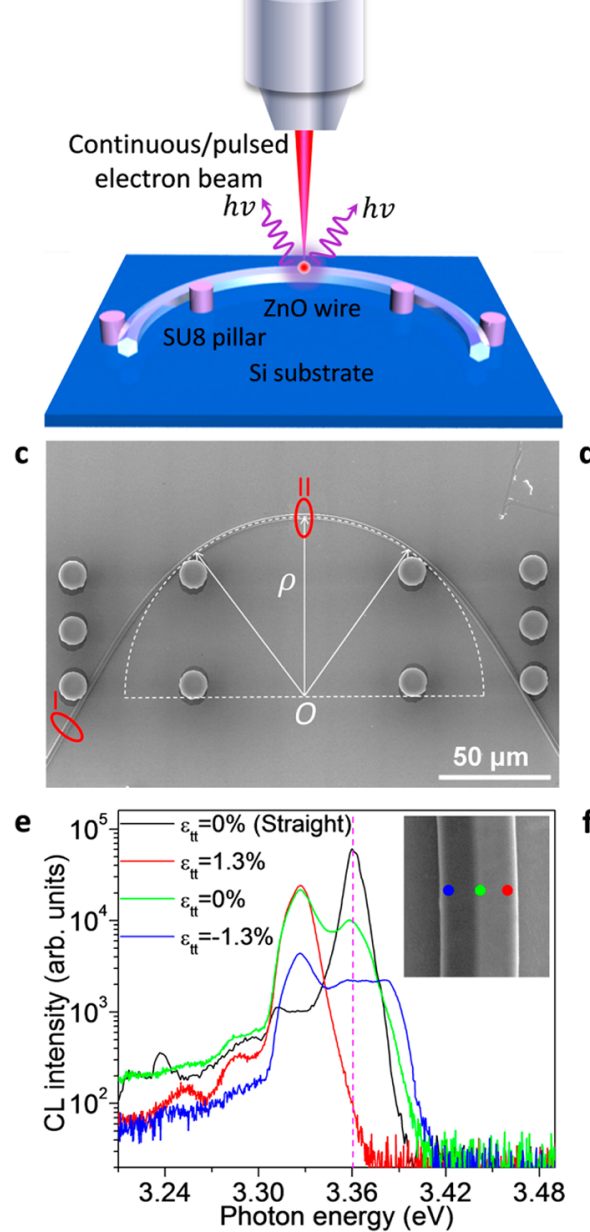

b

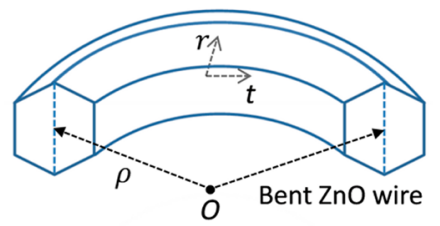

Neutral plane
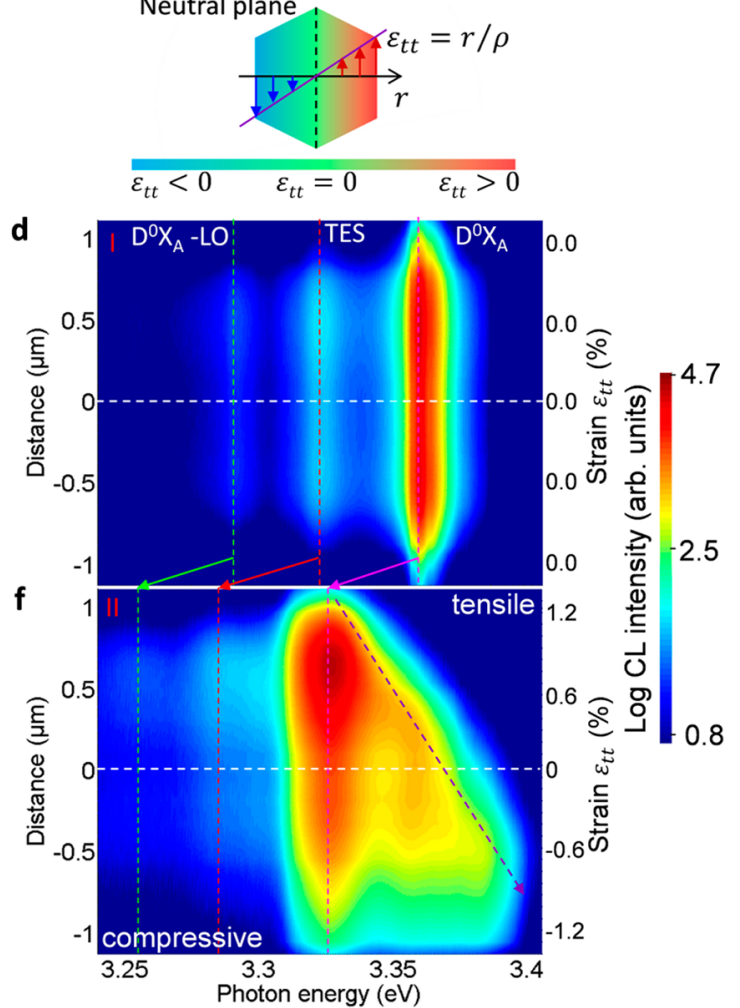

Figure 1. Schematic diagram for $\mathrm{CL}$ measurements on a purely bent $\mathrm{ZnO} \mathrm{MW}$ and low-temperature $\mathrm{CW}-\mathrm{CL}$ results. (a) Schematic diagram of CL measurements on the pure bending region of a 4PB ZnO MW. (b) Schematic figure of the local strain $\varepsilon_{\mathrm{tt}}$ distribution in the pure bending region of a 4PB ZnO MW (up) and the linear change of the local strain $\varepsilon_{\mathrm{tt}}$ in a purely bent cross section (down). The blue $\left(\varepsilon_{\mathrm{tt}}<0\right)$ and red $\left(\varepsilon_{\mathrm{tt}}>0\right)$ colors show the compressive and tensile regions, respectively. (c) SEM image of a typical standard 4PB ZnO MW with a diameter of $d=2.24 \mu \mathrm{m}$. $\rho$ shows the radius of curvature of the neutral plane in the purely bent region. ( $d$ and $\mathrm{f}$ ) Line-scanning $\mathrm{CW}-\mathrm{CL}$ spectra along a straight cross section "I" and a pure bending cross section "II" as indicated in part c. The geometric neutral plane of the wire is set as zero for the position axis. (e) CW-CL spectra at the straight cross section "I" and with an excitation spot outside $\left(\varepsilon_{\mathrm{tt}}=1.3 \%\right)$, in the middle of $\left(\varepsilon_{\mathrm{tt}}=0 \%\right)$, and inside $\left(\varepsilon_{\mathrm{tt}}=-1.3 \%\right)$ the bent section "II". The pink dashed line indicates the strain-free peak position of $D^{0} \mathrm{X}_{\mathrm{A}}$ luminescence.

To understand this difference and reveal the specificities of exciton dynamics in inhomogeneous strain fields, we further carried out investigations on this $4 \mathrm{~PB}$ ZnO MW using an original time-resolved $\mathrm{CL} \operatorname{setup}^{33}$ at $8 \mathrm{~K}$ (see Materials and Methods section).

Figure 2a presents the time-integrated CL spectra under pulsed electron excitation $(\sim 1 \mathrm{ps})$ for the straight segment "I" $\left(\varepsilon_{\mathrm{tt}}=0 \%\right)$ and three representative positions along the pure bending cross section "II": point $\mathrm{A}$ on the inside $\left(\varepsilon_{\mathrm{tt}}=-1.18 \%\right)$, point $\mathrm{B}$ in the middle $\left(\varepsilon_{\mathrm{tt}}=0 \%\right)$, and point $C$ on the outside $\left(\varepsilon_{\mathrm{tt}}=\right.$ $1.03 \%)$. The time-integrated spectra look similar to those obtained by CW-CL (Figure 1e), except for the change of the relative intensity of the high- and lowenergy peaks for excitation at the inner side. The corresponding streak-camera images for the timedependent $C L$ signals obtained from the straight segment and the points $A, B$, and $C$ from the compressive inside to the tensile outside along a cross section in the pure bending segment are presented in Figure $2 b-e$. Clearly, the streak-camera images at points $A, B$, and C are dramatically different from that of the straight segment. The most striking feature is the "comma shape" of these time-resolved $C L$ signals at the innermost point $A$ and central point $B$, which directly illustrates that the photon energy of the NBE luminescence undergoes a time-dependent red-shift after excitation. This time-dependent red-shift evidences a movement of excitons from high to low emission energy region (tensile outside), while no apparent exciton movement is observed after excitation at the outermost point $C$.

Figure 3 presents the time-dependent decay traces at different emission energies (left column) and $\mathrm{CL}$ spectra at different time delays (right column) extracted from the streak-camera images in Figure 2. In the decay traces corresponding to innermost point $A$ 

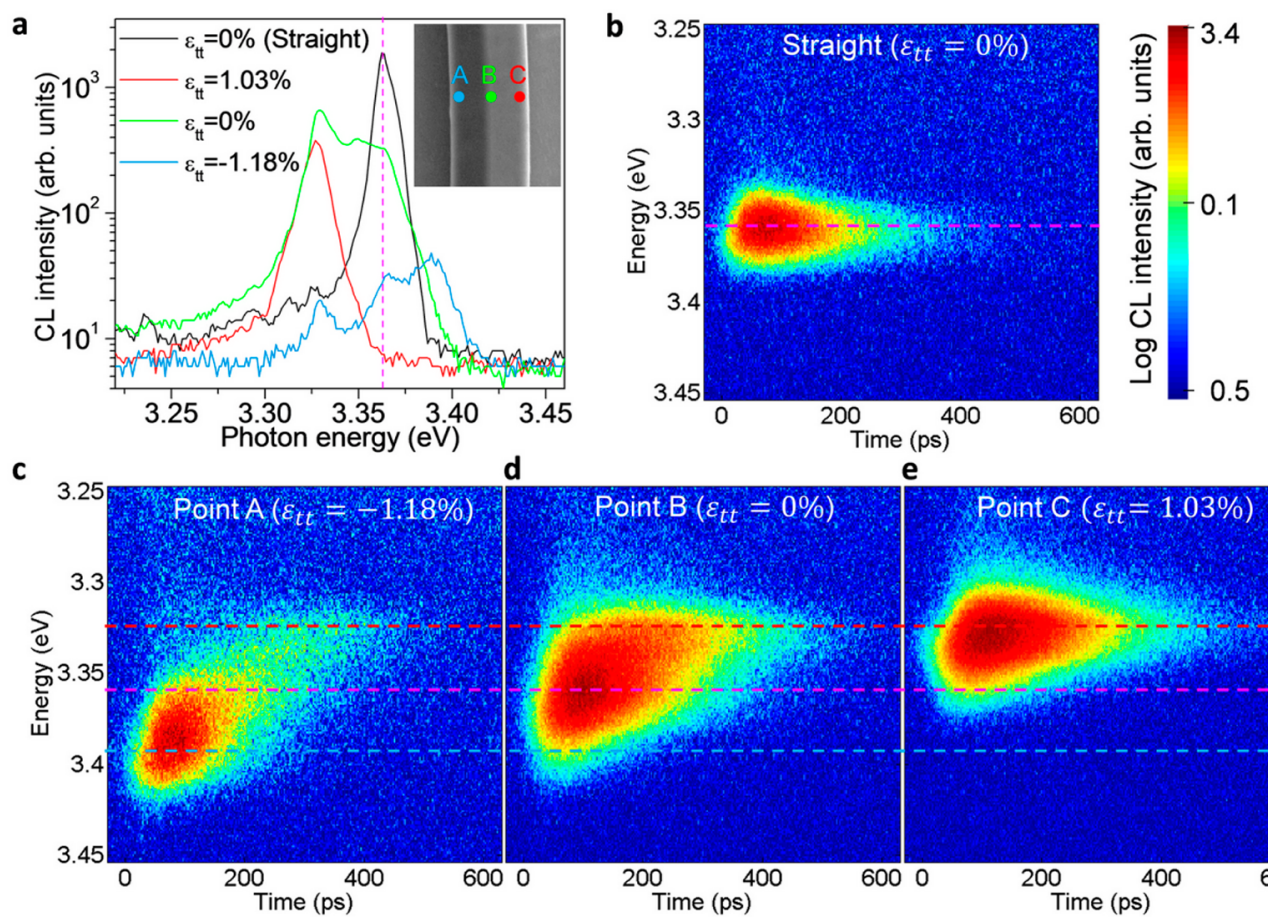

d Point $B\left(\varepsilon_{t t}=0 \%\right)$

e
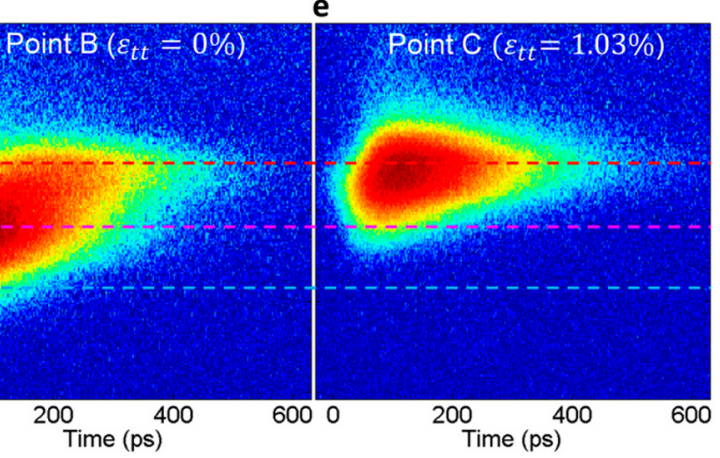

Figure 2. Time-resolved $\mathrm{CL}$ spectra results from both straight and pure bending segments at $8 \mathrm{~K}$. (a) Time-integrated $\mathrm{CL}$ spectra with pulsed electron beam exciting at points $\mathrm{A}$ (inside: $\varepsilon_{\mathrm{tt}}=-1.18 \%$ ), B (middle: $\varepsilon_{\mathrm{tt}}=0 \%$ ), and C (outside: $\varepsilon_{\mathrm{tt}}=1.03 \%$ ), as indicated in the inset, as well as the straight part. (b-e) Streak-camera images showing emission energy as a function of time (the intensity being color coded) after pulsed electron excitation the straight part (b) and points $A, B$, and $C$ of the purely bent section $(c, d, e)$. The pink dashed line indicates the strain-free peak position of $D^{0} X_{A}$ luminescence. The blue and red dashed lines show the emission photon energy of 3.392 and $3.322 \mathrm{eV}$, respectively.

(Figure 3a), the higher emission energy shows very short rise and decay times, while the lower emission energy has longer rise and decay times. The rise time $\tau_{\mathrm{r}}$ (the time for the signal to rise from $10 \%$ to $90 \%$ of the maximum value) and decay time $\tau_{\mathrm{d}}$ (fitted by firstorder exponential decay function) at $3.375 \mathrm{eV}$ are 60 and $70 \mathrm{ps}$, respectively, while the rise and decay times change to 90 and 100 ps at an intermediate energy of $3.358 \mathrm{eV}$, respectively. Most importantly, for the excitons at an even lower energy of $3.325 \mathrm{eV}$, the decay time $\tau_{\mathrm{d}}$ remains at about $100 \mathrm{ps}$, but the rise time $\tau_{\mathrm{r}}$

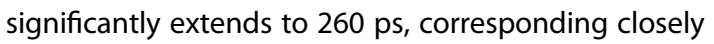
to the time needed for the high-energy luminescence to disappear completely. As seen clearly in Figure 3b, the emission peak gradually red-shifts from 3.392 to $3.322 \mathrm{eV}$, for time windows shifting from 90 to $410 \mathrm{ps}$ after excitation. It is worth noting that $3.322 \mathrm{eV}$ is precisely the exciton emission energy at the outer edge of the purely bent $\mathrm{ZnO} \mathrm{MW}$ (Figure 1f). The emission peak becomes increasingly broad with time, while a structure can be observed at about $3.365 \mathrm{eV}$ that becomes a peak at 280 ps (the origin of this structure will be discussed later). The behavior of both the decay traces and time-dependent spectra directly illustrate the donor-bound exciton dynamics in the pure bending cross section: the excitons formed upon e-beam excitation at the inside (with larger band gap) drift toward the outside (with smaller band gap) of the bent MW. Similar exciton dynamic features can be observed with excitation at the central point B (Figure $3 c$ and $d$ ). The rise and decay times at $3.365 \mathrm{eV}$ are 70 and $80 \mathrm{ps}$, respectively, and increase to 160 and $100 \mathrm{ps}$ at $3.325 \mathrm{eV}$. The time-dependent $\mathrm{CL}$ spectrum red-shifts from 3.365 to $3.322 \mathrm{eV}$ for time slots shifting from 100 to 500 ps. On the contrary, no obvious exciton drift can be observed at the outermost point $C$ (Figure 3e and f). Indeed, created at the energy minimum of the diameter, excitons do not have any possibility to shift along the strain gradient. The timedependent $C L$ spectrum only slightly red-shifts from 3.331 to $3.322 \mathrm{eV}$, and the rise and decay times at $3.325 \mathrm{eV}$ (70 and $100 \mathrm{ps)}$ ) are similar to those of the straight segment (Supplementary Figure S3).

In order to explain the observed bound exciton dynamics in this bent $\mathrm{ZnO}$ MW, a simple drift-diffusion mechanism taking into account the strain gradient is proposed. Basically, elastic strain in semiconductors results in the variation of the electronic band structure. ${ }^{7,25}$ As confirmed by our room-temperature CW-CL results, the NBE emission energy changes linearly with the local strain across the section of the bent segment. As a result, the generated excitons drift because of the change in band-gap energy along the strain gradient. Figure 4a schematically shows the one-dimensional exciton transport mechanism after excitation at the compressive side of the bent wire. Since we deal with donor-bound excitons in $\mathrm{ZnO}$ here, such a drift is unexpected by traditional theories. However, bound 

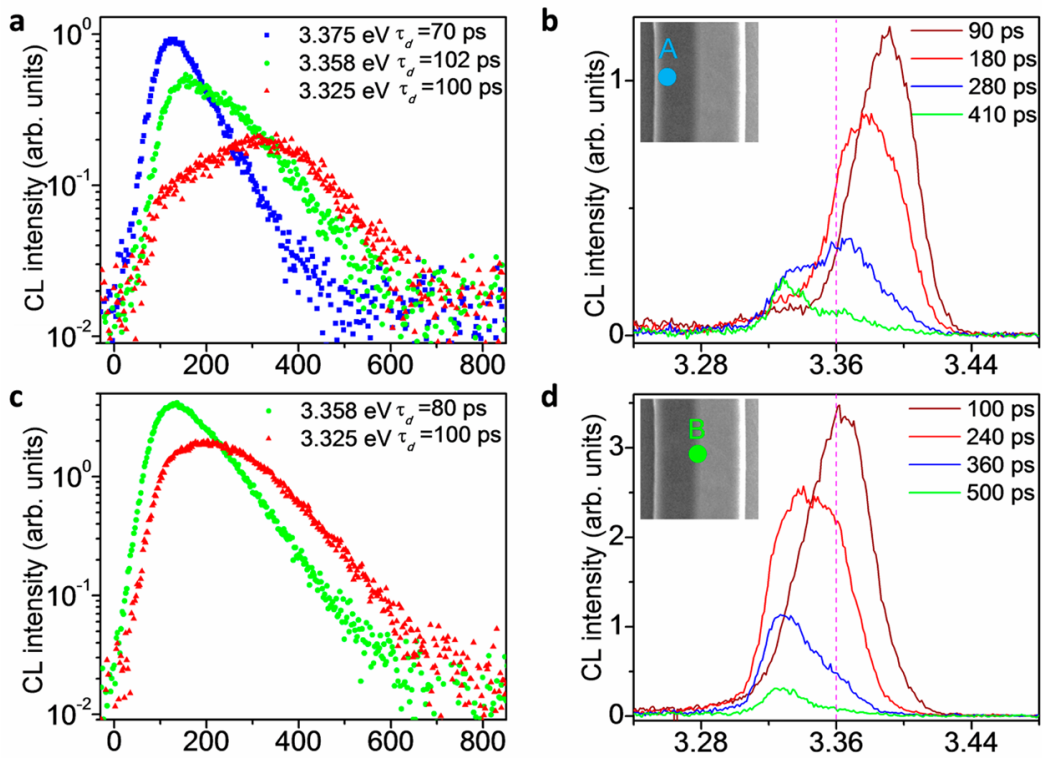

d
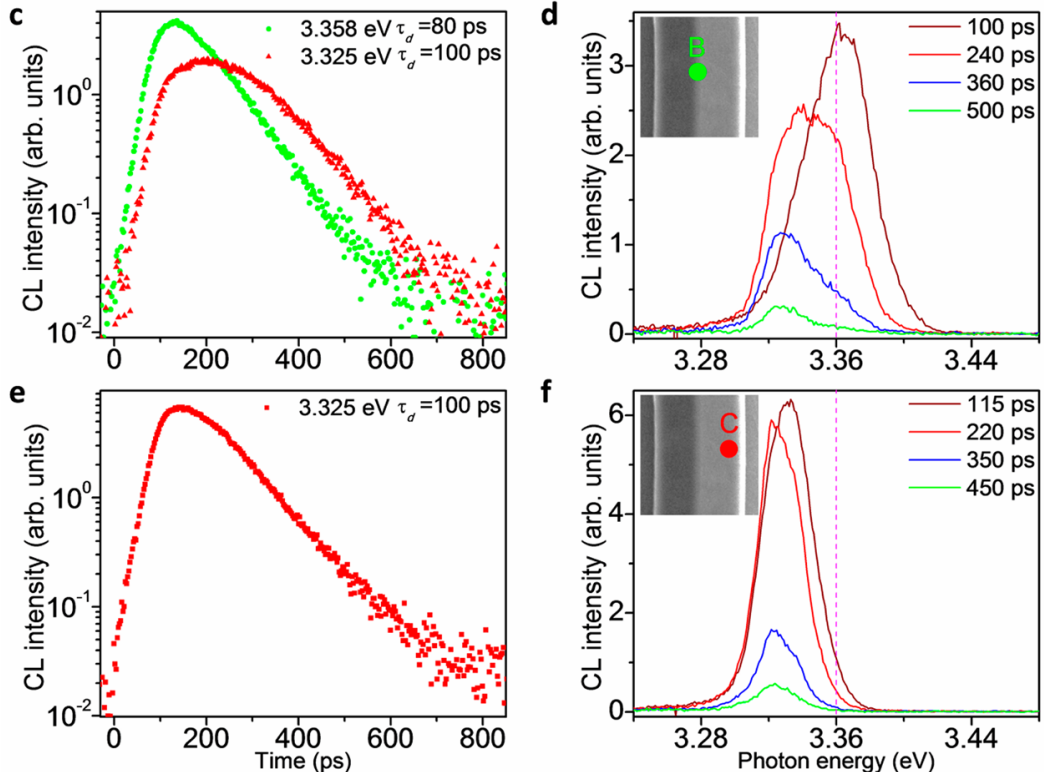

Figure 3. Time-dependent decays and time-resolved CL spectra at different time delays for excitation at the inside, middle, and outside of the purely bent section of the MW. The photon energies or the time delay are indicated in the figures. The first row $(a, b)$ corresponds to excitation at the inside of the MW (point $\left.A: \varepsilon_{t t}=-1.18 \%\right)$, the second row $(c, d)$ to the middle (point $B$ : $\varepsilon_{\mathrm{tt}}=0 \%$ ), and the third row $(\mathrm{e}, \mathrm{f})$ to the outside of the MW (point $\left.\mathrm{C}: \varepsilon_{\mathrm{tt}}=1.03 \%\right)$.

excitons can still move by hopping process from donor to donor. This mechanism for donor-bound exciton via phonon coupling has already been reported for nitrogen traps in GaAsP:N and GalnP:N. ${ }^{34}$ The hopping mechanism can be a very efficient process at low temperature, in particular at the donor densities existing in our wires, and can be described by an average exciton mobility for a given strain gradient. Further details on such a mechanism can be found in ref 35 . We favor hopping of the donor-bound excitons compared to the possible drift of free excitons, trapped onto donors along their drift along the strain field. Indeed, if we observe the spectrum of the straight part of the $\mathrm{ZnO}$ wire, the free exciton line appears only as a shoulder of the donor-bound exciton emission. This means that the capture process by donors is much faster than the radiative lifetime of excitons. In addition, our direct observation of a change with time of the donor-bound exciton spectrum is not compatible with the motionless donor-bound exciton. We indeed observe that the whole luminescence is shifted to low energies with time. This clearly favors the motion of donor-bound exciton even if we cannot discard a possible drift of free exciton. The excitonic potential shows a continuously varying profile, which follows the change of the band gap (the change of exciton binding energy under strain is neglected here). This results in an equivalent built-in field $E$ for the excitons, which can be written as

$$
E=\frac{\partial E_{\mathrm{ex}}(r)}{\partial r}=\frac{\partial E_{\mathrm{ex}}(r)}{\partial \varepsilon_{\mathrm{tt}}} \frac{\partial \varepsilon_{\mathrm{tt}}}{\partial r}
$$

where $E_{\mathrm{ex}}(r)$ is the excitonic potential within the cross section in the pure bending segment, $\left(\partial E_{\mathrm{ex}}(r)\right) / \partial \varepsilon_{\mathrm{tt}}=$ $-27.3 \mathrm{meV} / \%$ is the deformation potential of excitons, and $\partial \varepsilon_{\mathrm{tt}} / \partial \mathrm{r}=1 / \rho=1.25 \% \mu \mathrm{m}^{-1}$ is the uniform elastic strain gradient in the pure bending cross section. This equivalent built-in field $E$ for the excitons is unique, can only be formed in inhomogeneous strain fields, and is determined by the strain gradient.

After excitation at a given location within the cross section, the time-dependent drift-diffusion equation for the excitons can be written as

$$
\frac{\partial n(r, t)}{\partial t}=-\frac{n(r, t)}{\tau}+D \frac{\partial^{2} n(r, t)}{\partial r^{2}}-\mu E \frac{\partial n(r, t)}{\partial r}
$$

where $n(r, t)$ is the density of the excitons, $\tau$ is the recombination lifetime of the excitons (contains both 

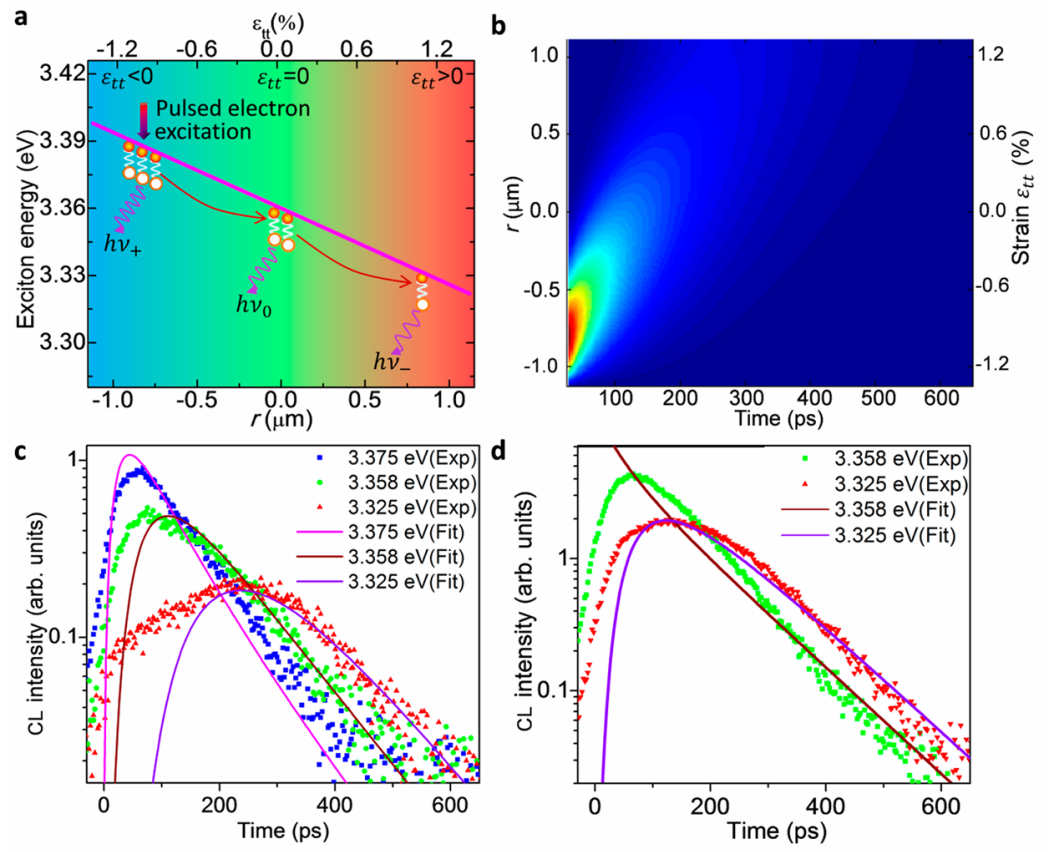

Figure 4. Schematic diagram of exciton drift mechanism in the purely bent $\mathrm{ZnO} \mathrm{MW}$ and drift-diffusion model simulation result. (a) Schematic diagram of donor-bound exciton drift mechanism in a cross section of the purely bent $\mathrm{ZnO} M W$ after pulsed electron excitation at the inner side (high-energy region). The blue $\left(\varepsilon_{\mathrm{tt}}<0\right)$ and red $\left(\varepsilon_{\mathrm{tt}}>0\right)$ colors show the compressive and tensile regions. (b) Drift-diffusion model simulation of the time-dependent exciton distribution in the $\mathrm{ZnO}$ MW pure bending cross section after pulsed excitation at the inner side (point $A$ ). ( $c$ and d) Best fits of the experimental decay traces at different photon energies with pulsed excitation at the innermost point $A$ and the central point $B$ in the pure bending cross section.

the radiative and nonradiative lifetime), $D$ is the exciton diffusion coefficient, $\mu$ is the average exciton mobility, and $E$ is the equivalent built-in field introduced in eq 1.

Figure $4 \mathrm{~b}$ shows the results, within the above model, of the time-dependent simulation of the exciton distribution across the MW after excitation at the innermost point $A$ (see Materials and Methods section). Clearly, due to the equivalent built-in field, the excitons drift toward the outside part of the bent MW, possessing a lower energy. This results in the time-dependent red-shift of the exciton emission peak, in agreement with the time-resolved $C L$ results in Figure $3 b$ and $d$. The decay traces at different emission energies are fitted reasonably well by this drift-diffusion model for exciting at both points $A$ and $B$ (Figure $4 c$ and d). Fitting of the experimental results allows us to deduce an exciton mobility of about $1400 \pm 100 \mathrm{~cm}^{2} \mathrm{eV}^{-1} \mathrm{~s}^{-1}$ for this bent $\mathrm{ZnO} \mathrm{MW}$. This value is in good agreement with the simple estimation we can made directly from the streak image of Figure 2c: It takes about 400 ps for an exciton to drift from the high-energy $(3.392 \mathrm{eV})$ side to the low-energy ( $3.322 \mathrm{eV}$ ) side. This time corresponds to the time for the exciton to travel $d \approx 1.8 \mu \mathrm{m}$ (distance from excitation spot to outside). We deduce an average speed of $5 \mathrm{~nm} / \mathrm{ps}$. In a gradient field of $E \approx 312.5 \mathrm{eV} / \mathrm{cm}$ (70 meV in $2.24 \mu \mathrm{m}$ ), this corresponds to an exciton mobility of about $1450 \mathrm{~cm}^{2} \mathrm{eV}^{-1} \mathrm{~s}^{-1}$, close to the value deduced from our drift-diffusion model. Hence, the time-resolved cathodoluminescence measurement provides a direct method to estimate exciton mobility in such a system.

The same drift-diffusion model explains also quite well the different $\mathrm{CW}-\mathrm{CL}$ results at low and room temperatures. At $8 \mathrm{~K}$, the generated excitons move quickly toward the outside of the MW, due to the high exciton mobility, before they recombine there. Only a small portion of the excitons recombines near the excitation site. Therefore, the line scanning CW-CL spectra show an intense red-shifted peak and a blue detuned weaker peak that shifts continuously when the excitation point moves toward the inside of the MW (Figure 1e and f). At room temperature, the larger phonon and defect scattering rates reduce the mobility of the excitons, nonradiative recombinations reduce their lifetime, and the exciton drift effect is no longer observable. The generated excitons recombine radiatively at the excitation location, resulting in a continuous shift of the NBE luminescence upon moving the excitation position across the section of the bent wire (Supplementary Figure S2).

The only observation that does not fit with our model is the additional peak at $3.365 \mathrm{eV}$ in the $\mathrm{CW}-\mathrm{CL}$ result at $8 \mathrm{~K}$, whose energy does not change when the excitation spot is moving to the inner compressive side. This peak is also observed in the time-resolved $C L$ spectra (Figure $2 a$ and Figure $3 b$ ). One possibility for the origin of this peak would be the strain-induced valence bands crossing, ${ }^{36}$ but our 
polarization measurements invalidate this assumption (Supplementary Figure S4). We tentatively attribute the additional peak to one of the whispering gallery modes (WGMs) that are known to exist in $\mathrm{ZnO}$ MWs. ${ }^{9,37}$ As WGMs are by nature coming from the entire diameter of the wire, they do not change with excitation positions, as confirmed by our $\mathrm{CW}-\mathrm{CL}$ result (Figure $1 \mathrm{f}$ ). However, since this peak is close to the band-gap energy, the refractive index is expected to show a strong dispersion and possible strong coupling could occur, ${ }^{38}$ which makes the simulation of the energy of the mode difficult to predict.

It has been previously suggested that the spatial distribution of the photoexcited carriers in bent $\mathrm{ZnO}$ NWs could be influenced by the transverse electric field associated with the piezoelectric effect. ${ }^{21} \mathrm{How}$ ever, the transverse piezoelectric field can influence the motion of only charged carriers, but has no effect on the motion of the neutral excitons in $\mathrm{ZnO}$, because of their strong binding energy. If the piezoelectric field would be strong enough to ionize the bound excitons into charged electrons and holes, they would move in opposite directions in space and no luminescence could be observed. Therefore, our investigation demonstrates that the donor-bound exciton drift induced by the elastic strain gradient is the reason of the overall energetic red-shift in bent $\mathrm{ZnO}$ micro/ nanowires observed in all previous works. ${ }^{17,20,21}$

\section{CONCLUSION}

In conclusion, through systematic investigations by $\mathrm{CW}$ - and time-resolved $\mathrm{CL}$ on purely bent $\mathrm{ZnO} \mathrm{MWs}$ with high spectral, spatial, and temporal resolutions at $8 \mathrm{~K}$, we find that the bound excitons can be driven by the elastic strain gradient toward the tensile outer side of the MW with lower exciton energy. A drift-diffusion model taking into account the strain gradient is proposed to describe the exciton dynamics in the inhomogeneous fields and can capture the main feature of the experimental results. The model allows us to deduce a mobility of $1400 \pm 100 \mathrm{~cm}^{2} \mathrm{eV}^{-1} \mathrm{~s}^{-1}$ for the donor-bound excitons in the bent $\mathrm{ZnO}$ wire. This novel strain gradient induced exciton drift in inhomogeneous strain fields should be general to most semiconductors. Our results therefore pioneer an innovative way to probe the exciton dynamics in semiconductors and might open the door for novel designs for optoelectronic and sensing micro/nanodevices.

\section{MATERIALS AND METHODS}

ZnO MWs Growth. The synthesis of ZnO MWs was carried out in a horizontal quartz tube furnace by chemical vapor deposition. The mixture of pure zinc oxide (99.9999\%) and graphite powder (molar ratio of 1:1) was loaded in an alumina boat. Sapphire chips with (110) orientation were placed above the source powder as the collecting substrates. The boat was then placed at the center of the quartz tube and inserted into a rapid heating furnace. The system was purged of contaminants with argon gas for more than $10 \mathrm{~min}$. After this, the growth carrier gas argon was maintained at $200 \mathrm{sccm}$ flow. The furnace was heated to $1050{ }^{\circ} \mathrm{C}$ in $20 \mathrm{~min}$, and then the oxygen $(3.0 \mathrm{sccm}$ ) was introduced as the reactive gas. The reaction proceeded for $30 \mathrm{~min}$, after which the system was cooled to room temperature. After this process the substrate is covered by a layer of $\mathrm{ZnO}$ MWs of high quality. All the ZnO MWs grow along the [0001] axis with a hexagonal cross-section.

Standard Four-Point-Bending Test. A series of SU8 (SU-8, 2015) pillar arrays, with a diameter of $6.0 \mu \mathrm{m}$ and a height of $15.0 \mu \mathrm{m}$, were fabricated on a Si substrate by photolithography and developing technology. The SU8 photoresist is spin coated on the Si substrate (500 rpm for $10 \mathrm{~s}$ and then $3000 \mathrm{rpm}$ for $60 \mathrm{~s}$ ) and heated $\left(65^{\circ} \mathrm{C}\right.$ for $1 \mathrm{~min}$ and then $95^{\circ} \mathrm{C}$ for $3 \mathrm{~min}$ ) until solidified, followed by UV lamp exposure $\left(12 \mathrm{~s}, 13 \mathrm{~mW} / \mathrm{cm}^{2}\right)$ with a predesigned mask template and developing with developer solution. After depositing a $20 \mathrm{~nm}$ Au layer by electron-beam evaporation deposition, an individual $\mathrm{ZnO} \mathrm{MW}$ is transferred from the growth substrate onto the prearranged substrate under an optical microscope by using two needle-shaped glass tips. The $\mathrm{ZnO} \mathrm{MW}$ is manipulated and positioned in the middle of four SU8 pillars, which make the wire take a curved shape after the end of the manipulation with the two glass tips. According to ASTM E855-08, in the standard 4PB setup, the bending part between the two inner SU8 pillars is expected to be under a pure bending state. Such bending deformation is elastic, and the curved microwire can resume its original straight state once it is taken out of the SU8 pillars.

Continuous-Wave CL Measurement. To obtain the optimum spatial resolution with best signal-to-noise ratio, an electron beam was accelerated at $10 \mathrm{kV}$ (spot size $4 \mu \mathrm{m}$, beam current of about $0.353 \mathrm{nA}$ ). It results, in $\mathrm{ZnO}$, in a generation volume of about $100 \mathrm{~nm}$ (with $90 \%$ power in this region, as supported by Monte Carlo simulation). ${ }^{39}$ The CL spectra were carefully collected step by step along the radial direction across the diameter of the $\mathrm{ZnO}$ microwires from the inner side to the outer side by $\mathrm{CL}$ spectroscopy (Gatan monocle $3+$ ) at liquid helium temperature $(8 \mathrm{~K})$. The $\mathrm{CL}$ spectra were recorded by a CCD (charge coupled device) with a scanning range of 300 to $450 \mathrm{~nm}$ with a spectral resolution of about $0.5 \mathrm{~nm}$. The line-scanning step size was set at $200 \mathrm{~nm}$ considering the typical exciton diffusion length is about $200 \mathrm{~nm}$ in $\mathrm{ZnO}$ at this temperature. ${ }^{40}$

Time-Resolved CL Measurement. The experimental setup for the time-resolved $\mathrm{CL}$ (TRCL) is composed of three blocks: the excitation block, the Attolight system for quantitative cathodoluminescence, and the detection block. The excitation block generates ultraviolet light pulses $(266 \mathrm{~nm}, 200 \mathrm{fs}, 80.7 \mathrm{MHz}$ repetition rate, spot size of $3 \mu \mathrm{m})$ using a mode-locked Ti: sapphire laser (Coherent Mira 900F, $800 \mathrm{~nm}, 1 \mathrm{~W}$ ) pumped by a continuous, high-power laser (Coherent Verdi V8, $532 \mathrm{~nm}$, $8 \mathrm{~W}$ ). A frequency tripler (Inrad M/N 5-050) is then used to achieve ultraviolet wavelengths. These ultraviolet light pulses are collimated into an optical fiber whose end is coated with a $20 \mathrm{~nm}$ thick gold ( $\mathrm{Au}$ ) layer, serving as a photocathode, to generate femtosecond electron pulses. Quantitative cathodoluminescence is performed using an Attolight Alalin Chronos 4027 system. The Attolight system integrates an achromatic reflective lens (NA 0.71) within the objective lens of the electron scanning microscope, thus aligning their focal planes and suppressing any necessary alignment. The Attolight system is also equipped with a specifically designed cryo-stage (drift $<10 \mathrm{~nm}$ ), easing measurements at low temperatures. The cathodoluminescence signal is diffracted spectrally by a threeturret grating monochromator (IHR 320) and collected by a synapse CCD camera. For the time-resolved signal, a streak camera (Hamamatsu C5680) with 2 ps resolution is used in the photon counting mode.

Drift-Diffusion Model Simulation. The drift-diffusion model based on the differential eq 2 has been solved by means of 
discretization with finite difference methods. The excitation of the system by a Gaussian beam of electrons (full width at halfmaximum (fwhm) of $50 \mathrm{~nm}$ ) has been taken as initial condition at time 0 . The parameters used for the simulation are $D$, the exciton diffusion coefficient $D=13 \mathrm{~cm}^{2} / \mathrm{s} ; \mu$, the exciton mobility $\mu=1400 \mathrm{~cm}^{2} \mathrm{eV}^{-1} \mathrm{~s}^{-1} ; \tau$, the effective exciton lifetime $\tau=300 \mathrm{ps}$; and $v_{\text {surf }}$, the surface recombination velocity, $v_{\text {surf }}=5 \times 10^{5} \mathrm{~cm} / \mathrm{s}$. In the simulation, the surface recombination velocity is implemented by adding a recombination term in eq 2 for the boundary conditions for $r=D / 2$ (surface of the wire): ${ }^{41}$

$$
\begin{aligned}
\frac{\partial}{\partial t} n\left(\frac{D}{2}, t\right) & =-\frac{1}{\tau} n\left(\frac{D}{2}, t\right)+D \frac{\partial^{2}}{\partial r^{2}} n\left(\frac{D}{2}, t\right) \\
& -\mu E \frac{\partial}{\partial r} n\left(\frac{D}{2}, t\right)-\frac{1}{\tau_{\text {surf }}} n\left(\frac{D}{2}, t\right)
\end{aligned}
$$

Since this surface coefficient is defined only for the last pixel, we define a surface recombination velocity, independent of discretization procedure:

$$
v_{\text {surf }}=\frac{\Delta r}{\tau_{\text {surf }}}
$$

where $\Delta r$ is the size of the discretization step.

By solving eq 2, we obtain the distribution of carrier along the wire as a function of time. Equation 1 and CW-CL measurements then allow us to get the relation between $r$ and the luminescence energy $E_{\mathrm{CL}}$. Since the $\mathrm{CL}$ intensity is proportional to the exciton density (we assume a constant radiative decay time along the wire), we obtained the CL spectra as a function of time with our model. In order to take into account the broadening of the cathodoluminescence spectra, we convolve $n(r)$ with a Gaussian function with a fwhm of $7 \mathrm{meV}$.

Conflict of Interest: The authors declare no competing financial interest.

Acknowledgment. This work is supported by the National 973 Program of China (2013CB932602, 2013CB921900), National Science Foundation of China (NSFC 11234001, 521 11174009) and National Project (MOST-11327902). The authors are also grateful for the financial support from the Sino-Swiss Science and Technology Cooperation Program (2010DFA01810) and NCCR Quantum Photonics. The authors want to thank Prof. Ju Li (MIT) and Z. L. Wang (GIT) for fruitful and comprehensive discussions.

Supporting Information Available: (Figure S1) SEM image of the $\mathrm{ZnO}$ microwires as grown. (Figure S2) Room-temperature continuous-wave $\mathrm{CL}$ results of the pure bent $\mathrm{ZnO}$ microwire. (Figure S3) Temporal-dependent decay trace and CL spectra at different time delays with pulsed electron excitation in the straight part. (Figure S4) Polarization characterizations of the $D^{0} X_{A}$ emission peak in the straight segment and the three emission peaks in the pure bending segment. This material is available free of charge via the Internet at http://pubs.acs.org.

\section{REFERENCES AND NOTES}

1. Hayden, O.; Agarwal, R.; Lieber, C. M. Nanoscale Avalanche Photodiodes for Highly Sensitive and Spatially Resolved Photon Detection. Nat. Mater. 2006, 5, 352-356.

2. Yan, R.; Gargas, D.; Yang, P. Nanowire Photonics. Nat. Photonics 2009, 3, 569-576.

3. Tian, B.; Zheng, X.; Kempa, T. J.; Fang, Y.; Yu, N.; Yu, G.; Huang, J.; Lieber, C. M. Coaxial Silicon Nanowires as Solar Cells and Nanoelectronic Power Sources. Nature 2007, 449, 885-889.

4. Garnett, E. C.; Yang, P. Silicon Nanowire Radial p-n Junction Solar Cells. J. Am. Chem. Soc. 2008, 130, 9224-9225.

5. Duan, X.; Huang, Y.; Agarwal, R.; Lieber, C. Single-Nanowire Electrically Driven Lasers. Nature 2003, 421, 241-245.

6. Tsukazaki, A.; Ohtomo, A.; Onuma, T.; Ohtani, M.; Makino, T.; Sumiya, M.; Ohtani, K.; Chichibu, S. F.; Fuke, S.; Segawa, Y.; et al. Repeated Temperature Modulation Epitaxy for p-Type Doping and Light-Emitting Diode Based on ZnO. Nat. Mater. 2004, 4, 42-46.
7. Feng, J.; Qian, X.; Huang, C.; Li, J. Strain-Engineered Artificial Atom as a Broad-Spectrum Solar Energy Funnel. Nat. Photonics 2012, 6, 866-872.

8. Guinea, F.; Katsnelson, M. I.; Geim, A. K. Energy Gaps and a Zero-Field Quantum Hall Effect in Graphene by Strain Engineering. Nat. Phys. 2009, 6, 30-33.

9. Fu, X.; Su, C.; Fu, Q.; Zhu, X.; Zhu, R.; Liu, C.; Liao, Z.; Xu, J.; Guo, W.; Feng, J. Tailoring Exciton Dynamics by Elastic Strain-Gradient in Semiconductors. Adv. Mater. 2014, 10.1002/adma.201305058.

10. leong, M.; Doris, B.; Kedzierski, J.; Rim, K.; Yang, M. Silicon Device Scaling to the Sub-10-nm Regime. Science 2004, 306, 2057-2060.

11. Minary-Jolandan, M.; Bernal, R. A.; Kuljanishvili, I.; Parpoil, V.; Espinosa, H. D. Individual GaN Nanowires Exhibit Strong Piezoelectricity in 3D. Nano Lett. 2012, 12, 970-976.

12. Wang, Z. L.; Song, J. Piezoelectric Nanogenerators Based on Zinc Oxide Nanowire Arrays. Science 2006, 312, 242246.

13. Zhu, T.; Li, J. Ultra-Strength Materials. Prog. Mater. Sci. 2010, 55, 710-757.

14. Wang, L.; Zheng, K.; Zhang, Z.; Han, X. Direct Atomic-Scale Imaging About the Mechanisms of Ultralarge Bent Straining in Si Nanowires. Nano Lett. 2011, 11, 2382-2385.

15. Signorello, G.; Karg, S.; Björk, M. T.; Gotsmann, B.; Riel, H. Tuning the Light Emission from GaAs Nanowires over 290 meV with Uniaxial Strain. Nano Lett. 2013, 13, 917-924.

16. Wei, B.; Zheng, K.; Ji, Y.; Zhang, Y.; Zhang, Z.; Han, X. SizeDependent Bandgap Modulation of ZnO Nanowires by Tensile Strain. Nano Lett. 2012, 12, 4595-4599.

17. Han, X.; Kou, L.; Lang, X.; Xia, J.; Wang, N.; Qin, R.; Lu, J.; Xu, J.; Liao, Z.; Zhang, X.; et al. Electronic and Mechanical Coupling in Bent ZnO Nanowires. Adv. Mater. 2009, 21, 49374941.

18. Dietrich, C. P.; Lange, M.; Klüpfel, F. J.; Wenckstern, H. von; Schmidt-Grund, R.; Grundmann, M. Strain Distribution in Bent ZnO Microwires. Appl. Phys. Lett. 2011, 98, 031105.

19. Xue, H.; Pan, N.; Li, M.; Wu, Y.; Wang, X.; Hou, J. G. Probing the Strain Effect on Near Band Edge Emission of a Curved ZnO Nanowire via Spatially Resolved Cathodoluminescence. Nanotechnology 2010, 21, 215701.

20. Han, X.; Kou, L.; Zhang, Z.; Zhang, Z.; Zhu, X.; Xu, J.; Liao, Z.; Guo, W.; Yu, D. Strain-Gradient Effect on Energy Bands in Bent ZnO Microwires. Adv. Mater. 2012, 4707-4711.

21. Xu, S.; Guo, W.; Du, S.; Loy, M.; Wang, N. Piezotronic Effects on the Optical Properties of ZnO Nanowires. Nano Lett. 2012, 12, 5802-5807.

22. Fu, Q.; Zhang, Z. Y.; Kou, L.; Wu, P.; Han, X.; Zhu, X.; Gao, J.; $\mathrm{Xu}$, J.; Zhao, Q.; Guo, W.; et al. Linear Strain-Gradient Effect on the Energy Bandgap in Bent CdS Nanowires. Nano Res. 2011, 4, 308-314.

23. Sun, L.; Kim, D. H.; Oh, K. H.; Agarwal, R. Strain-Induced Large Exciton Energy Shifts in Buckled CdS Nanowires. Nano Lett. 2013, 13, 3836-3842.

24. Wang, Z. L. Progress in Piezotronics and Piezo-Phototronics. Adv. Mater. 2012, 24, 4632-4646.

25. Bardeen, J.; Shockley, W. Deformation Potentials and Mobilities in Non-Polar Crystals. Phys. Rev. 1950, 549.

26. Tamor, M.; Wolfe, J. Drift and Diffusion of Free Excitons in Si. Phys. Rev. Lett. 1980, 44, 1703-1706.

27. Trauernicht, D.; Wolfe, J.; Mysyrowicz, A. Highly Mobile Paraexcitons in Cuprous Oxide. Phys. Rev. Lett. 1984, 52, 855-858.

28. Fu, X.; Liao, Z.; Liu, R.; Xu, J.; Yu, D. Size-Dependent Correlations between Strain and Phonon Frequency in Individual ZnO Nanowires. ACS Nano 2013, 7, 8891-8898.

29. ASTM E855-08. Standard Test Methods for Bend Testing of Metallic Flat Materials for Spring Applications Involving Static Loading; ASTM International, 2013, DOI: 10.1520/ E0855-08R13, www.astm.org

30. Hamby, D. W.; Lucca, D. A.; Klopfstein, M. J.; Cantwell, G. Temperature Dependent Exciton Photoluminescence of Bulk ZnO. J. Appl. Phys. 2003, 93, 3214.

31. Meyer, B. K.; Alves, H.; Hofmann, D. M.; Kriegseis, W.; Forster, D.; Bertram, F.; Christen, J.; Hoffmann, A.; 
Straßburg, M.; Dworzak, M.; et al. Bound Exciton and Donor-Acceptor Pair Recombinations in ZnO. Phys. Status Solidi B 2004, 241, 231-260.

32. Rowe, J.; Cardona, M.; Pollak, F. Valence Band Symmetry and Deformation Potentials of ZnO. Solid State Commun. 1968, 6, 239.

33. Merano, M.; Sonderegger, S.; Crottini, A.; Collin, S.; Renucci, P.; Pelucchi, E.; Malko, A.; Baier, M. H.; Kapon, E.; Deveaud, B.; et al. Probing Carrier Dynamics in Nanostructures by Picosecond Cathodoluminescence. Nature 2005, 438, 479-482.

34. Mariette, H.; Kash, J.; Wolford, D.; Marbeuf, A. Exciton Transfer at Low Temperature in $\mathrm{Ga}_{-}\{\mathbf{x}\} \operatorname{In} \_\{1-\mathrm{x}\} \mathrm{P:N}$ and GaAs_\{1-x\}P_\{x\}:N. Phys. Rev. B 1985, 31, 5217-5222.

35. Jacopin, G.; Shahmohammadi, M.; Ganière, J.-D.; Deveaud, B. Hopping Process of Bound Excitons under an Energy Gradient. Appl. Phys. Lett. 2014, 104, 042109.

36. Liao, Z.-M.; Wu, H.-C.; Fu, Q.; Fu, X.; Zhu, X.; Xu, J.; Shvets, I. V; Zhang, Z.; Guo, W.; Leprince-Wang, Y.; et al. Strain Induced Exciton Fine-Structure Splitting and Shift in Bent $\mathrm{ZnO}$ Microwires. Sci. Rep. 2012, 2, 452.

37. Nobis, T.; Kaidashev, E.; Rahm, A.; Lorenz, M.; Grundmann, M. Whispering Gallery Modes in Nanosized Dielectric Resonators with Hexagonal Cross Section. Phys. Rev. Lett. 2004, 93, 103903.

38. Trichet, A.; Durupt, E.; Médard, F.; Datta, S.; Minguzzi, A.; Richard, M. Long-Range Correlations in a 97\% Excitonic One-Dimensional Polariton Condensate. Phys. Rev. B 2013, $88,121407$.

39. Drouin, D.; Couture, A. R.; Joly, D.; Tastet, X.; Aimez, V.; Gauvin, R. CASINO V2.42: A Fast and Easy-to-Use Modeling Tool for Scanning Electron Microscopy and Microanalysis Users. Scanning 2007, 29, 92-101.

40. Yoo, J.; Yi, G.-C.; Dang, L. S. Probing Exciton Diffusion in Semiconductors Using Semiconductor-Nanorod Quantum Structures. Small 2008, 4, 467-470.

41. Lambert, B.; Clerot, F.; Deveaud, B.; Chomette, A.; Talalaeff, G.; Regreny, A.; Sermage, B. Electron and Hole Transport Properties in GaAs-AlGaAs Superlattices. J. Lumin. 1989, $44,277-283$. 